\title{
La relación entre la distancia del lanzamiento de pelota por lo alto y la velocidad máxima de la pelota del saque en los jugadores de tenis juniors elite
}

\author{
Károly Dobos y Csaba Nagykáldi
}

\author{
Palabras clave: velocidad de \\ la pelota, correlación, \\ movimientos pliométricos
}

Recibido: 14 Mayo 2017

Aceptado: 27 Agosto 2017

Autor correspondiente:

Károly Dobos.

Correo electrónico:

doboskaresztenisz@gmail.com

\section{INTRODUCCIÓN}

En el juego moderno de tenis, el rol clave del saque es indudable. El saque es el único elemento técnico que el jugador ejecuta independientemente de la pelota del oponente. Esta ejecución independiente asegura el mayor nivel posible de control de movimiento (Bhamonde \& Knudson, 2003). Además, la capacidad de los jugadores en generar pelotas de alta velocidad se ha vuelto uno de los fundamentos básicos de rendimiento exitoso en competición (Cross \& Pollards, 2009). El golpeo del saque con una mayor velocidad provoca un tiempo de preparación más corto para el receptor. Con un segundo saque con una velocidad media de $117 \mathrm{~km} / \mathrm{hora}$, el tiempo de preparación es de 1,200 milisegundos, y disminuye hasta 900 milisegundos en el caso de un primer saque con una velocidad de $160 \mathrm{~km} / \mathrm{hora}$. El tiempo pasado en preparación disminuye aún más en canchas duras, es decir 200 milisegundos (Kleinöder, 2005). A nivel profesional, no es extraño que las pelotas de saque vuelen a una velocidad de $200 \mathrm{~km} /$ hora par los hombres y $190 \mathrm{~km} / \mathrm{hora}$ para las mujeres. Este resulta en una mayor disminución en el tiempo de preparación, por eso se piensa que la utilización de material de entrenamiento desarrollando las capacidades motrices (por ejemple: el lanzamiento de la pelota por lo alto) en la fase de preparación (espacialmente para los jugadores de tenis juniors) tiene una importancia clave, porque ayuda el jugador en formar movimientos de saque adecuados y en acelerar la raqueta.

Reid, Giblin \& Whiteside (2015) y Wagner et al. (2014) investigaron sobre la relación entre las especialidades cinemáticas del lanzamiento por lo alto y el saque. Los resultados mostraron que el lanzamiento por lo alto y la velocidad del saque indican una ligera correlación positiva. Aparte de los similitudes mecánicas de los dos movimientos, hay algunas diferencias mecánicas que muestran la diferencia entre los dos tipos de movimientos. Pero, los investigadores están de acuerdo que el modelo de movimiento del lanzamiento por lo alto asegura una base adecuada para desarrollar el saque.

Las pruebas mencionadas se realizaron con solamente una pequeña muestra $(n=28 ; n=10)$. Además, la relación que existe entre la distancia del lanzamiento de la pelota por lo alto y la velocidad máxima del saque también puede ser interesante como sugerencias por parte de los profesionales y de los investigadores ya demostraron la relación entre la velocidad del golpe y la distancia de los diferentes lanzamientos (Genevois, Pollet, Rogowski, 2014; Ikeda et al., 2007). Además, el objetivo de esa investigación en una larga muestra 
representativa conteniendo ambos sexos (80 chicos y 80 chicas) era probar la hipótesis que la distancia del lanzamiento de la pelota por lo alto de los jugadores de tenis juniors elite $y$ la velocidad del saque muestra una correlación positiva cercana en ambos sexos.

\section{MÉTODOS Y PROCEDIMIENTOS}

La muestra se constituye de jugadores de tenis juniors elite (menos de 12, 14, 16 y 18 años). Los sujetos fueron seleccionados con el método de muestra aleatoria estratificada, entonces en cada grupo de edad hay 20 chicos y 20 chicas, 160 personas en total fueron probadas. Del punto de vista de la investigación, se forman un grupo de edad de chichos y uno de chicas (Tabla 1). La muestra seleccionada representa las mejores poblaciones de chicas y chicos de los jugadores de tenis juniors húngaros.

Para examinar la relación entre la velocidad máxima de la pelota del saque y la distancia del lanzamiento de la pelota por lo alto, se utilizaron dos ensayos de campo (Figure 1.) (Nádori et al., 2005; Ulbricht, Fernandez-Fernandez \& Ferrauti, 2013). Los jugadores tenían que ejecutar los ensayos de campo en un orden dado (lanzamiento de pelota por lo alto y velocidad del saque) después de 15 minutos de calentamiento clásico. Los jugadores tenían tres ensayos por las pruebas del lanzamiento por lo alto y ocho por ellas de la velocidad del saque. Los mejores resultados fueron utilizados en la análisis. La pequeña pelota pesaba 100 gramos (diámetro $6.5 \mathrm{~cm}$ ). Para medir la velocidad del saque, se utilizó el medidor de velocidad "Stalker ATS II" (con una precisión dentro de $1 \mathrm{~km} / \mathrm{h}$ ).

\begin{tabular}{|c|c|c|c|c|c|}
\hline \multirow[t]{2}{*}{$\operatorname{sen} 0$} & \multirow{2}{*}{$\begin{array}{l}\text { Edad } \\
\text { Media(SD) }\end{array}$} & \multicolumn{2}{|c|}{$\begin{array}{l}\text { Distuncia del lanzuniento } \\
\text { de la pelota por lo also (m) }\end{array}$} & \multicolumn{2}{|c|}{$\begin{array}{l}\text { Velosidad matuina de hi } \\
\text { pelota on sque (kmh) }\end{array}$} \\
\hline & & Gama (SD) & Ranpe & Media (SD) & Gama \\
\hline \multirow[t]{2}{*}{ Chicus } & $14.37(2.24)$ & 28.84 & 1657 . & 140.61 & 87.00. \\
\hline & & $(6.07)$ & 4430 & (19.12) & 176.00 \\
\hline \multirow[t]{2}{*}{ Chices } & 14.3012 .22 & 41.83 & 25.75 . & 157.56 & 110.00 . \\
\hline & & (9.4l) & 6618 & (22.97) & 21100 \\
\hline
\end{tabular}

Tabla 1. Estadísticas básicas en los jugadores de tenis juniors elite.
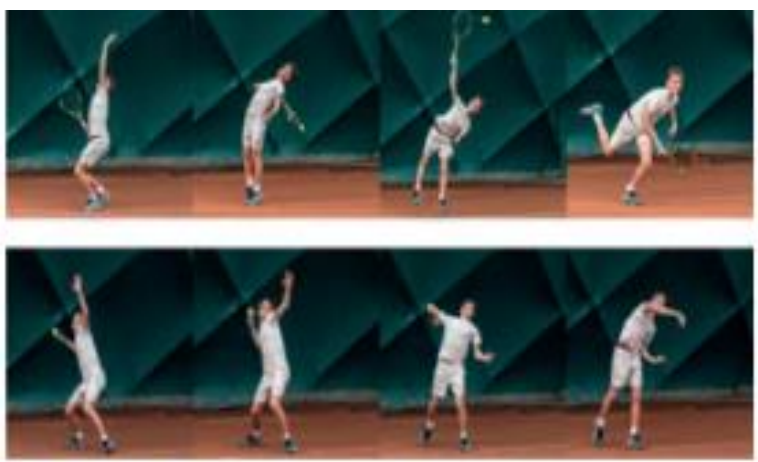

Figura 1. Pruebos de saque y del lanzamiento de la pelota por lo alto
El coeficiente de correlación Pearson se calculó para determinar la relación entre la velocidad máxima de la pelota en el saque y la distancia alcanzada con el lanzamiento de la pelota por lo alto. El nivel de significancia se determinó en $p<$ 0.05. El análisis estadístico de datos fue llevado a cabo con el programa SPSS 13.0

\section{RESULTADOS}

En ambos sexos, una fuerte correlación positiva fue encontrada entre la distancia del lanzamiento de la pelota por lo alto y la velocidad máxima de la pelota del saque (Figura 2. 3.).

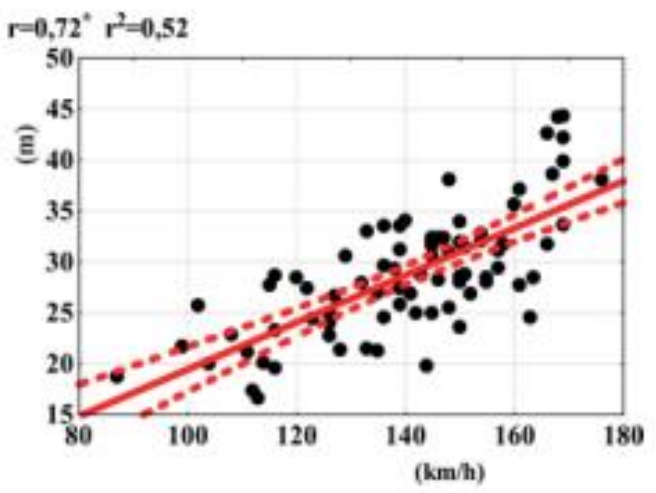

Figura 2. Coeficientes de correlación entre la distancia del lanzamiento de la pelota por lo alto y la velocidad máxima de la pelota del saque en las jugadoras de tenis juniors elite $p^{*}(0.05$.

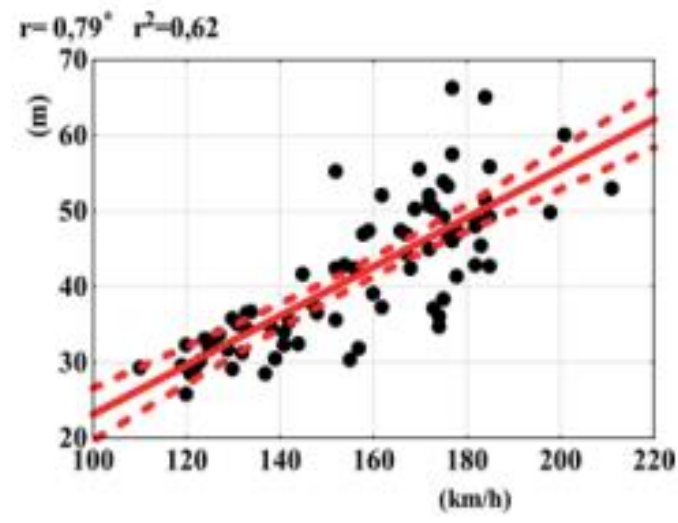

Figura 3. Coeficientes de correlación entre la distancia del lanzamiento de la pelota por lo alto y la velocidad máxima de la pelota del saque en los jugadores de tenis juniors elite $p^{\star}$ co.os.

\section{DEBATE}

La fuerza explosiva del brazo superior dominante de los jugadores de tenis juniors elite mostraba una fuerte correlación positiva con la velocidad máxima de la pelota en el saque. Los resultados demuestran claramente la idea que la 
llamada forma de movimientos "pliométricos" (el ciclo extender - acortar) es el tipo de contracción de músculo más frecuente en el tenis, dado que el modelo de coordinación de la mayoría de los golpes contiene este tipo de contracción. Así, los jugadores de tenis que puedan usar sus fuerzas con más eficacidad son capaces de golpear la pelota más fuerte, y tendrán los saques más fuertes (Chu, 2003). Además, las similitudes mecánicas de estos ambos movimientos (Reid, Giblin \& Whiteside, 2015; Wagner et al., 2014) ayudan al jugador en desarrollar un saque exitoso. Por lo tanto, según nuestra opinión, la fuerza explosiva presente en el lanzamiento de la pelota por lo alto puede transferirse en modelo de movimiento del saque.

Por eso, el uso del lanzamiento de la pelota por lo alto como práctica de lanzamiento pliométrica es imprescindible para crear una velocidad de saque adecuada. Además, los coeficientes decisivos también muestran (chicas, $r 2=0.52$; chicos, r2=0.62) que las dos formas de movimientos tienen similitudes, pero no son idénticas. Los resultados refuerzan las propuestas de los resultados de la investigación previa (Reid, Giblin \& Whiteside, 2015; Wagner et al., 2014).

\section{CONCLUSIONES}

Se llevó a cabo la investigación en una muestra representativa conteniendo todos los grupos de edad oficiales, en los cuales la distancia del lanzamiento de la pelota por lo alto y la velocidad máxima de la pelota del saque mostraron una fuerte y relevante correlación en ambos sexos. Por consiguiente, se sugiera que el lanzamiento de la pelota por lo alto sea una parte de la preparación general de los jugadores de tenis juniors y que se use como herramienta para identificar el rendimiento.

\section{REFERENCIAS}

Bhamonde, R., Knudson, D. (2003). Linear and angular momentum in stroke production. In Elliot, B, Reid, M., Crespo, M. (Eds.), Biomechanics of advanced Tennis (pp. 51-70). London: International Tennis Federation.

Chu, D. (2003). Increasing power in tennis. In: Reid, M., Quinn, A., \& Crespo, M. (Eds.), Strength and Conditioning for Tennis (pp. 137147). London: International Tennis Federation.

Cross, R., \& Pollard, G. (2009). Grand Slam men's single tennis 19912009 Serve Speeds and other related data. ITF Coaching and Sport Science Review, 16, 8-10.

Genevois, C., Pollet, T., \& Rogowski, I. (2014). Relationship between the performance of the forehand groundstroke and the onehand or two-hand medicine ball throw. ITF Coaching and Sport Science Review, 62 21-23. https://doi.org/10.52383/itfcoaching.v23i66.140
Ikeda, Y., Miyatsuji, K., Kawabata, K., Fuchimoto, T., \& Ito, A. (2009). Analysis of trunk muscle activity in the side medicine ball throw. Journal of Strength and Conditioning Research, 23, 2231-2240. https://doi.org/10.1519/JSC.0b013e3181b8676f

Kleinöder, H. (2005): The return of the serve. Retrieved May 20, 2010, from http: // www.coachesinfo.com/category/tennis.

Nádori, L., Derzsy, B., Fábián, Gy., Ozsváth, K., Rigler, E., \& Zsidegh, M. (2005). Sportképességek mérése (3nd ed.). [Measuring Sport Ability. In Hungarian] Budapest: Semmelweis University Faculty of Physical Education and Sport Sciences.

Reid, M., Giblin, G., \& Whiteside, D. (2015). A kinematic comparison of the overhand throw and tennis serve in tennis players: How Similar are they really? Journal of Sport Sciences, 33, 713-723. https://doi.org/10.1080/02640414.2014.962572

Ulbricht, A., Fernandez-Fernandez, J. \& Ferrauti, A. (2013). Conception for Fitness Testing and individualized training program in the German Tennis Federation. Sports Orthopaedics and Traumatology, 29, 180-192. https://doi.org/10.1016/j.orthtr.2013.07.005

Wagner, H., Pfusterschmied, J., Tilp, M., Landlinger, J., Duvillard von S.P., Müller, E. (2014). Upper body kinematics in team-handball throw, tennis serve and volleyball spike. Scandinavian Journal of Medicine \& Science in Sports. 24, 345-354. https://doi.org/10.1111/j.1600-0838.2012.01503.x

\section{CONTENIDO ITF ACADEMY RECOMENDADO (HAZ CLICK ABAJO)}

\section{ITF Academy}

Derechos de Autor (c) 2017 Károly Dobos y Csaba Nagykáldi

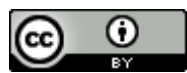

Este texto está protegido por una licencia CreativeCommons 4.0 .

Usted es libre para Compartir -copiar y redistribuir el material en cualquier medio o formato- y Adaptar el documento - remezclar, transformar y crear a partir del material- para cualquier propósito, , incluso para fines comerciales, siempre que cumpla la condición de:

Atribución: Usted debe dar crédito a la obra original de manera adecuada, proporcionar un enlace a la licencia, e indicar si se han realizado cambios. Puede hacerlo en cualquier forma razonable, pero no de forma tal que sugiera que tiene el apoyo del licenciante o lo recibe por el uso que hace de la obra.

Resumendelicencia - Textocompletodelalicencia 